\title{
Experimental Setup to capture high resolution images for Quality Control of GEM Foils
}

\section{C.A Rodriguez ${ }^{1}$, R.M Gutierrez}

CICBA - Centro de Investigaciones en Ciencias Básicas y Aplicadas

Universidad Antonio Nariño. Cra. 3 Este No. 47 A-15, Bloque 4 -Piso 3

Bogotá D.C., Colombia

E-mail: cesararodriguezQuan.edu.co

This work presents an experimental setup to capture high-resolution images of GEMfoils developed at the Detector's Laboratory at Antonio Nariño University. The setup consists of one digital camera with 18 megapixel CMOS aptina sensor attached to one 30 x 30 centimeters dual axis linear stepper to capture the high-resolution images. We used the high-resolution images to determine defects and geometry changes of the micro-holes contained in the GEM-foil using Software for Foils Analysis. The results show that using this setup, and the software is possible, identify variations in the geometry of the micro-holes of a GEM-foil up to one pixel $=1.25 \mu \mathrm{m}$. The automation of capture and image analysis improve the present quality control of GEM foils in accuracy and cost reduction. 


\section{Introduction}

The GEM-foils are an essential element of Micro Pattern Gaseous Detectors, MPGDs, which are state of the art of the radiation detectors. Diverse experiments of high energy physics use GEM-foils due to the extraordinary quality that increases the number of applications in different industrial and medical sectors [1,2]. A GEM-foil is a drilled sandwich structure composed of three layers (copper, Kapton, copper) with a density of 5, 50 and 5 microns respectively. The micro-holes are perforated and distributed all over the film by different methods, however, diverse defects can be found, despite of the technique used to perforate the GEM-foils. The Fig. 1 shows a representation of a cross section of a biconical hole; the size of the perforations is $70 \mu \mathrm{m}( \pm 5 \mu \mathrm{m})$ for both layers of copper and $50 \mu \mathrm{m}( \pm 5 \mu \mathrm{m})$ for the Kapton layer.

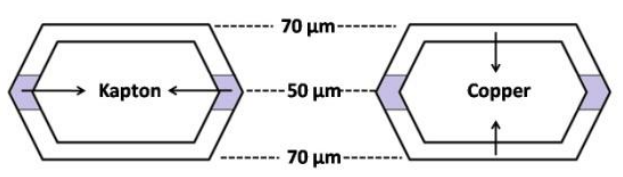

Figure 1: Cross section of a biconical hole

Due to the fact, the holes are in the range of micrometers in diameter, it is necessary to use highresolution image analysis. For this reason we developed an inexpensive setup to capture highresolution images of GEM-foil with quality control purposes. The measurement and detection of the objects in an image depend on the quality of the image and these in turn depend on the technology of the light sensors or detectors installed in the camera. In the case of a standard digital camera, it has an active pixel sensor where small photo-cells capture the reflected light from the structure to be reconstructed on an image [3]. The image's resolution depends on the optical lenses and the number of photocells that uses the camera to cover an area of the structure; the resolution is expressed in pixels [3]. The number of pixels is the resolution of the image and it is calculated multiplying the number of pixels of one row by the number of pixels of one column of the image [3]. In this work we used an OMAX Digital Camera for Microscope with an Aptina Image sensor; the maximum resolution is $4912 \times 3684$ pixels and its pixel size is about of 1.25 um x 1.25 um (Fig.2a).

\section{Experimental Method}

We used 2 cameras for the different test; for one of these experiments we used a 14 mega pixel camera with an HDMI port (Fig 2b) to record sequentially all the foil and thus are able to analyze it faster. We attached the monocular lens (Fig 2c) with $300 \mathrm{X}$ magnification to the cameras, because these devices are not able to reach micrometric sizes. We worked with the minimum distance, around 4.1 inches between the lens and the GEM-foil to take the images. 
a)

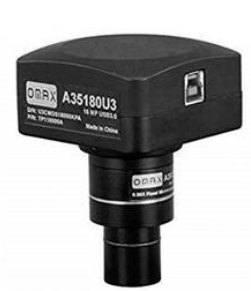

b)

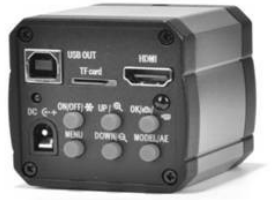

c)

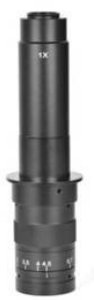

Figure 2: Optical Devices. a) 18 MP OMAX Digital Camera [4] b) 14MP AIHOME Camera c) Generic Monocular lens with $300 \mathrm{X}$ magnification

Finally, for the sequential taking of the images, we attached with high-precision screws the camera to the linear stepper (Fig.3) and align it with a laser system. The linear stepper has a precision of 1 micrometer per step. The range of the stepper is about of 12 inch x 12 inch and its speed is about of 1.5 meters per second. The maximum load capacity is 5 pounds.

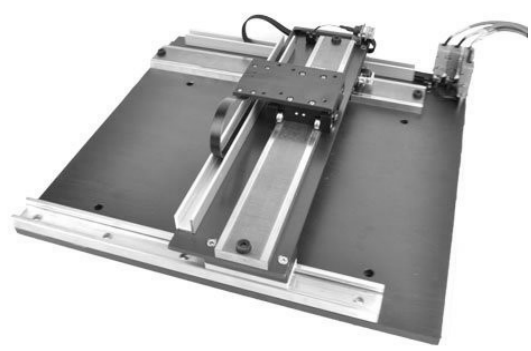

Figure 3: Linear stepper

\subsection{Results and Discussions}

The figure 6 presents the first image obtained using the setup. The image was captured manually and its resolution is about $5000 \times 4000$ pixels approximately that cover an area of $0.5 \mathrm{~mm}$ by 0.5 $\mathrm{mm}$ on the GEM-foil.

a)

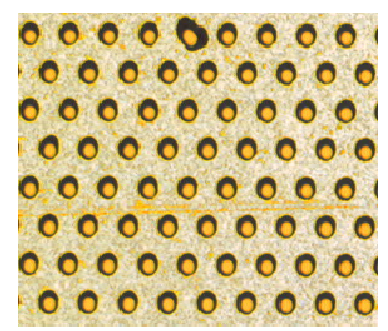

b)

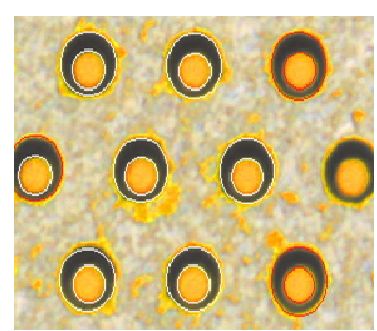

Figure 4: Image Analysis. a) Image resolution of 5000 pixels x 4000 pixels approx. b) Defect detected by SOFA The defective holes are highlighted in red and good holes in white. 
We analyzed the image using the Software (SOFA) developed at the detector's laboratory at Antonio Nariño University. The figure $4 \mathrm{~b}$ shows defects identified by SOFA using the inner radius size.

Although the images have a better resolution, we need to generate a synchronization process between the camera's shutter and the speed of the device. The Software offers an effective solution to lighting variations on the GEM-foil image; however, we have to explore different light sources with the purpose to improve the results.

\section{Conclusions}

This work presents an experimental setup to capture high-resolution images with quality control purposes. The results demonstrate that our software and the setup proposed are compatible to detect defects in GEM-Foils using high-resolution images. Therefore, the presented software and the setup are a not expensive and faster alternative to the current GEM-foil quality control processes. The software could help to support a R\&D process to correlate the performance of GEM detectors with GEM-foil quality using any similar setup.

\section{Outlook}

Our perspectives are to detect defects online using video inspection with machine learning techniques to reduce time and resources. In addition, we are working in the developing a portable prototype that can be used in large areas of GEM-foils.

\section{Acknowledgements}

We would like to express thanks to Administrative Department of Science, Technology and Innovation (COLCIENCIAS) for financing this work.

\section{References}

[1] F. Sauli, The gas electron multiplier (GEM): Operating principles and applications. 805.

[2] Tamm, B. P, Test and evaluation of new GEMs with an automatic scanner. Volume 556, Issue 2, 15 January 2006, Pages 527-534.

[3] J. F. Pertasu, Técnicas de análisis de imagen, Aplicaciones en Biología. Valencia, Universitat de Valencia, 2010.

[4] OMAX INC. Omax 18.0mp Usb3.0 Digital Camera For Microscope With 0.01mm Calibration Slide, http://www.omaxmicroscope.com 